\begin{tabular}{|c|l|}
\hline Title & Evaluation of the IRI model using CHA MP observations in polar and equatorial regions \\
\hline Author(s) & Liu, Huixin; Stolle, Claudia; Rother, Martin; W atanabe, Shigeto; A be, Takumi; Cooke, David L. \\
\hline Citation & $\begin{array}{l}\text { Advances in Space Research, 39(5), 904.909 } \\
\text { https://doi.org/10.1016/.asr.2006.08.006 }\end{array}$ \\
\hline Issue Date & 2007 \\
\hline Doc URL & http://hdl.handle.net/2115/32750 \\
\hline Type & article (author version) \\
\hline File Information & IRI05-16-watanabe_revised.pdf \\
\hline
\end{tabular}

Instructions for use 


\title{
Evaluation of the IRI Model Using CHAMP Observations in Polar and Equatorial Regions
}

\author{
Huixin Liu ${ }^{a}$, Claudia Stolle ${ }^{\mathrm{b}}$, Martin Rother ${ }^{\mathrm{b}}$, \\ Shigeto Watanabe ${ }^{\mathrm{a}}$, Takumi Abe ${ }^{\mathrm{c}}$, David L. Cooke ${ }^{\mathrm{d}}$ \\ ${ }^{\mathrm{a}}$ Earth and Planetary Science Division, Hokkaido University, Sapporo 060-0810, \\ Japan. \\ ${ }^{\mathrm{b}}$ GeoForschungsZentrum Potsdam, Telegrafenberg, 14473 Potsdam, Germany \\ c Japan Aerospace Exploration Agency (JAXA) , Sagamihara, Kanagawa 229-8510, \\ JAPAN \\ d Air Force Res. Lab., Hanscom AFB, MA 01731, USA
}

\begin{abstract}
This study compares the electron density and temperature observed by the CHAMP satellite and those predicted by the International Reference Ionosphere (IRI) model. The comparison has revealed a general agreement between CHAMP and IRI in the seasonal variation of the electron density and temperature. However, in polar regions, the model tends to overestimate the electron density and underestimate the electron temperature. In addition, the CHAMP observes a weaker winter anomaly in the southern hemisphere than in the northern hemisphere, while the IRI predictions are similar in both hemispheres. In the equatorial region, the model describes the dayside equatorial ionization anomaly fairly well, but underestimates its depth in the post-sunset local time sector by about $50 \%$. In polar regions, two prominent spatial structures observed by CHAMP are found to be missing in the IRI prediction.
\end{abstract}


Namely, a band of low electron density along the nightside auroral region and a band of elevated electron temperature near the cusp. The high-Te band near the cusp exhibits clear seasonal variation, with the largest latitude and local time coverage in summer and the smallest in winter.

Key words: International Reference Ionosphere, Polar Ionosphere, Equatorial Ionosphere

\section{Introduction}

The International Reference Ionosphere (IRI) is an important empirical model for the ionosphere, widely used by both theoretician and experimentalists (Bilitza, 2001). It describes reasonably well the quiet-time climatology of the electron density and temperature at middle latitudes. However, the model predictions often deviate from observations to a rather large degree at both equatorial and high latitudes. Therefore, further evaluation and improvement of the model using observations are needed in these regions.

The CHAMP (CHAllenging Minisatellite Payload) satellite was launched on 15 July 2000 into a near-circular orbit with an inclination of $87.3^{\circ}$ (Reigber et al., 2002). From its initial altitude of $456 \mathrm{~km}$, it has decayed to about $350 \mathrm{~km}$ after five years. The high inclination of the orbit ensures an almost complete latitudinal coverage in both hemispheres. In addition, the orbit plane precesses through all local times every four months. These configurations enable the CHAMP satellite well suited for the ionospheric/thermospheric studies in a global scale as demonstrated by several studies (e.g. Liu et al., 2005; Stolle et

Email address: huixin@ep.sci.hokudai.ac.jp (Huixin Liu). 
al., 2006).

The present work focuses on the seasonal and geomagnetic variation of the electron density and temperature in both polar (including auroral) and equatorial regions. Through a detailed comparison of the CHAMP observations and the predictions from the IRI model, we aim to contribute to the evaluation and improvement of the model in these regions.

\section{CHAMP measurements and the IRI configuration}

CHAMP uses a Planar Langmuir Probe (PLP) to measure the in-situ electron density $(\mathrm{Ne})$ and temperature (Te) every $15 \mathrm{~s}$. The accuracy of the PLP measurements is within 10\%. Our analysis is based on the PLP data from 1 January 2001 to 1 August 2004. To investigate the seasonal and geomagnetic variation, measurements of $\mathrm{Ne}$ and Te were normalized to a fixed solar flux level of F10.7=150 and to a fixed height of $400 \mathrm{~km}$ using the IRI model. By doing so, we have assumed that the model represents reasonably well the variation of the F2-region electron density and temperature with height and solar flux. The normalized data are then binned and averaged over magnetic local time (MLT) and geomagnetic latitudes with a $1 \mathrm{~h} \times 1^{\circ}$ (latitude) resolution. To assure statistical significance, measurements such as $\mathrm{Ne} \geq 5 \cdot 10^{6} \mathrm{~cm}^{-3}$ or Te $\geq 8000 \mathrm{~K}$ are first discarded before averaging. Then, a constraint of one $\sigma$ is set when calculating the mean values so that they represent frequently observed values instead of extreme ones.

The IRI model 2000 version is used in the present study. The storm module (Fuller-Rowell et al., 2000) was not activated, since its effect is mainly 
restricted in the mid-latitudes. Model predictions were generated for every sample point of the measurements at $400 \mathrm{~km}$ altitudes. However, the model was run at a fixed solar activity $(\mathrm{F} 10.7=150)$ to isolate the seasonal and geomagnetic variations. The predictions are subsequently binned and averaged in the same way as that for the measurements.

\section{Comparisons in Polar Regions}

This section presents the model-measurement comparison in high latitudes, for both the electron density and temperature. The comparison is described for magnetically quiet $(\mathrm{Kp}=0 . .2)$ conditions. The CHAMP observations for moderately disturbed $(\mathrm{Kp}=3 . .4+)$ conditions are also presented for possible future improvement of the IRI model. The seasons mentioned in the text are local seasons.

\subsection{Under Quiet Conditions}

\subsubsection{The Electron Density}

Figure 1 presents the electron density at $400 \mathrm{~km}$ altitude in different seasons obtained from CHAMP and IRI. Seasonal variation is shown in both CHAMP and IRI. On the dayside northern hemisphere $(\mathrm{NH})$ below about $75^{\circ} \mathrm{N}$, the electron density is almost double in winter than in summer. This shows that

the winter anomaly extends actually to latitudes as high as the cusp, rather than confined in the mid-latitudes (e.g. Kelley, 1989; Mendillo et al., 2005). The nightside Ne is highest in summer and lowest in winter, hence showing no winter anomaly. It merely reflects the change of solar zenith angle. The 
differences between IRI and CHAMP are mainly seen in two aspects. First, the CHAMP Ne is generally about $15 \%$ lower than the IRI predictions. Second, a band of low $\mathrm{Ne}$ in the nightside auroral region, i.e., along $60^{\circ}-65^{\circ} \mathrm{N}$ latitudes, is evident in the observations for all seasons except for summer. This feature is indiscernible in the IRI predictions.

In the southern hemisphere $(\mathrm{SH})$, large differences exist between the observations and model predictions. First on the dayside, CHAMP shows lower Ne in winter than in other seasons, hence observing no winter anomaly in the southern hemisphere above $50^{\circ} \mathrm{S}$. This is consistent with other observations showing a weaker winter anomaly in SH than in NH (Yonezawa , 1971; Mendillo et al., 2005). In contrast, the model produces a winter anomaly similar to that in the northern hemisphere. Second, a density trough exists in the CHAMP observations near 17 MLT around equinoxes, and 15 MLT in summer below about $65^{\circ} \mathrm{S}$. This feature is not reflected by the model. Third, a band of low Ne in the auroral region is discernible in the observations, but not in the model predictions. Fourth, the CHAMP Ne is generally lower than the predicted values. The difference reaches almost $30 \%$ in winter.

\subsubsection{The electron temperature}

Figure 2 presents the electron temperature at $400 \mathrm{~km}$ altitude in different seasons for the northern hemisphere. Features observed in the SH are similar, hence not shown here. Both CHAMP and IRI show a seasonal variation of Te, with maximum values in summer and minimum values in winter. However, the CHAMP Te appears to be $50 \%$ higher than the IRI values on average. A prominent difference exists in the spatial structure. CHAMP observes a 
band of elevated Te near the dayside cusp. The temperature inside the band is about $1000 \mathrm{~K}$ higher than that in adjacent regions. This band of enhanced Te is located between $\sim 74^{\circ}-80^{\circ} \mathrm{N}$ geomagnetic latitudes and in the 06-16 MLT sector around equinoxes. In summer, it expands and covers latitudes $\sim$ $72^{\circ}-82^{\circ} \mathrm{N}$ between $04-18 \mathrm{MLT}$. In winter, however, it is confined to a very narrow stripe along $76^{\circ}$ between $08-14$ MLT. Therefore, this local structure shows a clear seasonal variation, with the largest latitude and local time coverage in summer, and the smallest in winter. In comparison, the IRI shows no enhanced Te near the cusp. The Akebono satellite (Tsuruda and Oya , 1993) also observed these localized regions around cusp as shown in Figure 3, though the temperature tends to be higher than the CHAMP-observed values. Because of additional heat source in the high latitude region, the thermal electron detector on Akebono sometimes measures the energy distribution which can not be considered as Maxwellian. In such a case, the temperature tends to be overestimated. Hence, the averaged distribution shown here may represent somewhat higher temperatures overall. Nevertheless, the feature of local Te enhancement near the cusp region is evident.

\subsection{Under Disturbed Conditions}

In this section, we describe prominent features observed by the CHAMP satellite under moderately disturbed conditions. Since the IRI model does not include the storm module for the high latitude region, it is not surprising that it cannot reproduce localized structures presented below. 


\subsubsection{The electron density}

The seasonal variation is similar to that under quiet conditions. However, CHAMP observes two new features in the spatial distribution of the disturbedtime $\mathrm{Ne}$ in all seasons and in both hemispheres. Figure 4 shows the local autumn distribution as an example. A pronounced density maximum exists near $70^{\circ} \mathrm{N} / \mathrm{S}$ geomagnetic latitudes near local noon. In addition, the band of low Ne in the auroral region becomes more prominent than that under quiet conditions.

\subsubsection{The electron temperature}

The seasonal variation and spatial structure of the electron temperature are similar to the quiet-time ones. However, CHAMP observes a more pronounced region of enhanced Te near the cusp than under quiet conditions. The spring pattern is shown in Figure 5 as an example. In comparison to quiet conditions, the region of high Te expands with increasing geomagnetic activity both equator- and night-ward. In addition, the electron temperature increases as well. Polarward expansion is not observed. Similar Te behavior was found by Proelss (2005) in the DE2 observations .

\section{Comparisons in equatorial regions}

We focus on the Equatorial Ionization Anomaly (EIA) in this section, since it is one of the most important ionospheric structures in the equatorial region. As the CHAMP flies at about $400 \mathrm{~km}$ altitude, the latitude variation of the electron density it observes reflects to a large extent the EIA structure. Figure 
6 presents the case for March Equinox under different geomagnetic conditions. The upper row shows the latitudinal variation on the dayside between 11-15 MLT, and the lower row shows the one in the post-sunset local time sector (19-22 MLT). We see that the IRI reproduces the observed EIA on the dayside quite well, except for an underestimation of the crest-density under disturbed conditions. For the post-sunset EIA, however, IRI underestimates the crest values, but overestimate that at the equator, hence leading to a density trough which is about only half depth of the CHAMP observed one.

\section{Discussion}

The comparison has revealed a general agreement between CHAMP and IRI in the seasonal variation of the electron density and temperature. However, in polar regions, the model tends to predict electron density about 15-20\% higher and electron temperature 20-50\% lower than the CHAMP-observed values. Since these differences are larger than the accuracy of the CHAMP measurements (10\%), we may state that the IRI model overestimates the electron density, but underestimates the electron temperature in polar regions. In addition, the model predicts symmetric patterns for the southern and northern hemispheres, while the CHAMP observes rather asymmetric patterns. This leads to large differences in the southern hemisphere both in magnitude and in structures. In particular, the winter anomaly of the electron density shown by the model is similar in both hemispheres. However, the CHAMP observes a weaker winter anomaly in the $\mathrm{SH}$ than in the $\mathrm{NH}$, consistent with other observations. In the equatorial region, the model describes the dayside EIA fairly well. But it underestimates the depth of the post-subset EIA by about 
$50 \%$.

The CHAMP observations show two prominent spatial structures which are missing in the IRI predictions. The first one is a band of low electron density on the nightside along latitudes between about $50^{\circ}-60^{\circ}$. It is observed in all seasons except in summer, and becomes more pronounced with increasing geomagnetic activity. Given the location of this structure, it is very likely to be the mid-latitude trough. Trough formation is mainly due to plasma decay in regions of stagnation or chemical and dynamical processes associated with fast plasma motion in the rest frame of the neutral particles (see the review of Rodger et al., 1992, and references therein). Processes like ion outflows (Anderson et al., 1991; Liu et al., 2001) and strong vibrationally excited $N_{2}$ (Mishin et al., 2004) may also rise to further deplete the ionosphere under disturbed conditions. Furthermore, Rodger et al. (1992) has pointed out that an anti-correlation normally exists between the electron temperature and density in trough regions caused by stagnation or sub-auroral ion drift (SAID). However, neither CHAMP nor IRI reveals any clear signature of Te enhancement in the band of low electron density. We cannot find a clear explanation for this. Since features obtained in our study are statistically averaged, over many single cases and longitudes, it is possible that narrow bands of enhanced Te related to stagnation or SAID type of trough would be smoothed out during this process unless the enhancement is particularly large in a wide area. In addition, it is also possible that the Te measurement from CHAMP becomes less accurate when the electron density is too low.

The second structure is a band of elevated electron temperature centered near the cusp. It exhibits clear seasonal variation, with the largest latitude and local time coverage in summer and the smallest in winter. Expansion of this 
region is also observed at higher geomagnetic activity level. Soft particle precipitation has been shown to be efficient for F-region electron heating through direct ionization and its resultant photoelectrons (Abe et al., 1993; Liu et al., 1995). In addition, current-driven instabilities, i.e., Buneman instability, ion cyclotron and ion acoustic instability could also be important heating source. Forme et al. (1993) has shown that such instabilities seeded by intense fieldaligned currents (FAC) over a few tens of $\mu \mathrm{A} \mathrm{m} \mathrm{m}^{-2}$ can cause strong electron heating. Small-scale FAC (sFAC) over $100 \mu \mathrm{A} \mathrm{m} \mathrm{m}^{-2}$ has been frequently observed by CHAMP in the bands of high electron temperature (see Figure 7). Interestingly, the region of sFAC also exhibits a seasonal variation with larger spatial coverage in summer than in winter, and expands under disturbed geomagnetic conditions. These variations resemble that of the Te enhancement, hence could indicate possible contribution to the electron heating by strong current-driven instabilities.

Acknowledgements This work is supported by the JSPS research grant. We appreciate the operational support of the CHAMP mission by the German Aerospace Center (DLR).

\section{References}

Abe, T., Oyama, K.-I., Watanabe, S., Fukunishi, H., Characteristic features of electron temperature and density variations in field-aligned current regions, J. Geophys. Research, 88, 11,257-11,266, 1993.

Anderson, P. C., Heelis, R. A., Hanson, W. B. The ionospheric signatures of rapid subauroral ion drifts, J. Geophys. Research, 96, 5785-5792, 1991.

Bilitza, D., International Reference Ionophere 2000, Radio Sci., 36(2), 261- 
$275,2001$.

Forme, F. R. E., Wahlund, J. E., Opgenoorth, H. J., Persson, M. A. L., Mishin, E. V., Effects of current driven instabilities on the ion and electron temperature in the topside ionosphere, J. Atmos. and Solar-Terres. Physics, 55, 647-666, 1993.

Fuller-Rowell, T. J., Araujo-Pradere, E., Codrescu, M., An empirical ionospheric storm-time correction model, Adv. Space Res., 25(1),139-148, 2000.

Kelley, M., The earth's ionosphere, Academic press, 1989.

Liu, C., Horwitz, J. L., Richards, P. G., Effects of frictional ion heating and soft-electron precipitation on high-latitude F-region upflows, Geophys. Res. Lett., 22, 2713-2716, 1995.

Liu, H., Ma, S. Y., Schlegel, K., Diurnal, seasonal and geomagnetic variations of large field-aligned ion upflows in the high-latitude ionospheric F region, J. Geophys. Res., 106, 24,651-24,662, 2001.

Liu, H., Lühr, H., Henize, V., Köhler, W., Global distribution of the thermospheric total mass density derived from CHAMP, J. Geophys. Res., doi:10.1029/2004JA010741, 2005.

Mishin, E. V., Burke, W. J., Viggiano, A. A., Stormtime subauroral density troughs: Ion-molecule kinetics effects, J. Geophys. Res., doi:10.1029/2004JA010438, 2004.

Mendillo, M., Huang, C.-L., Pi, X., Rishbeth, H., Meier, R., The global ionospheric asymmetry in total electron content, J. Atmos. and Solar-Terres. Physics, 67, 1377-1387, 2005.

Proelss, G., Electron temperature enhancement beneath the magnetospheric cleft. Ann. Geophy., submitted, 2005.

Reigber, C., Lühr, H., Schwintzer, H., CHAMP mission status, Adv. Space Res., 30, 129-134, 2002. 
Rodger, A. S., Moffett, R. J., Quegan, S., The role of ion drift in the formation of ionisation troughs in the mid- and high-latitude ionosphere - a review, J. Atmos. and Solar-Terres. Physics, 54, 1-30, 1992.

Stolle, C., Lühr, H., Rother, M., Balasis, G., Magnetic signatures of equatorial spread F, as observed by the CHAMP satellite, J. Geophys. Res., doi:10.1029/2005JA011184, 2006.

Tsuruda, K., Oya, H., Introduction to the Akebono EXOS D project, J. Geophys. Res., 98, 11123-11126, 1993.

Yonezawa, T., The solar-activity and latitudinal characteristics of the seasonal, non-seasonal and semi-annual variations in the peak electron densities of the F2-layer at noon and at midnight in the middle and low latitudes, J. Atmos. and Solar-Terres. Physics, 33, 887-907, 1971. 


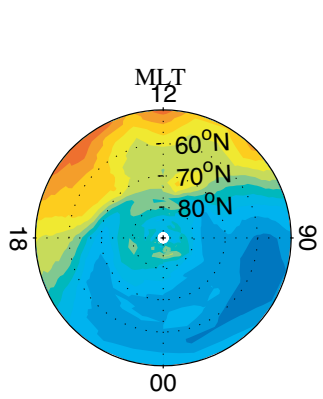

$\mathrm{Ne}$ NH CHAMP

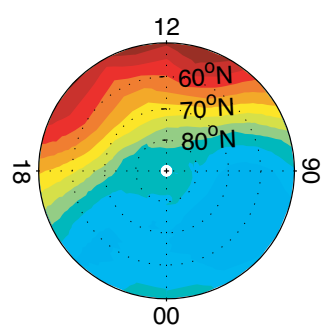

Ne NH IRI

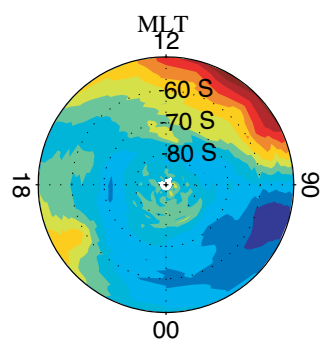

Ne SH CHAMP

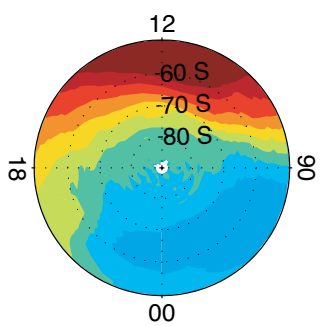

Ne SH IRI

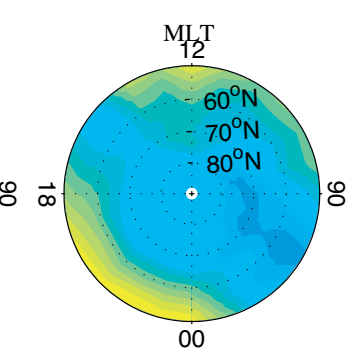

$\mathrm{Ne}$ NH CHAMP

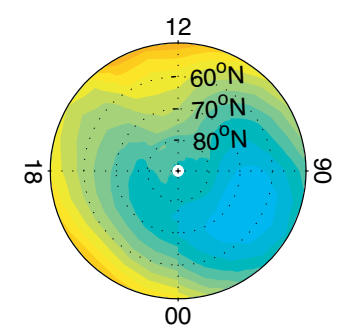

Ne NH IRI

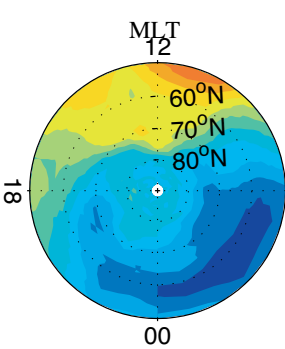

Ne NH CHAMP
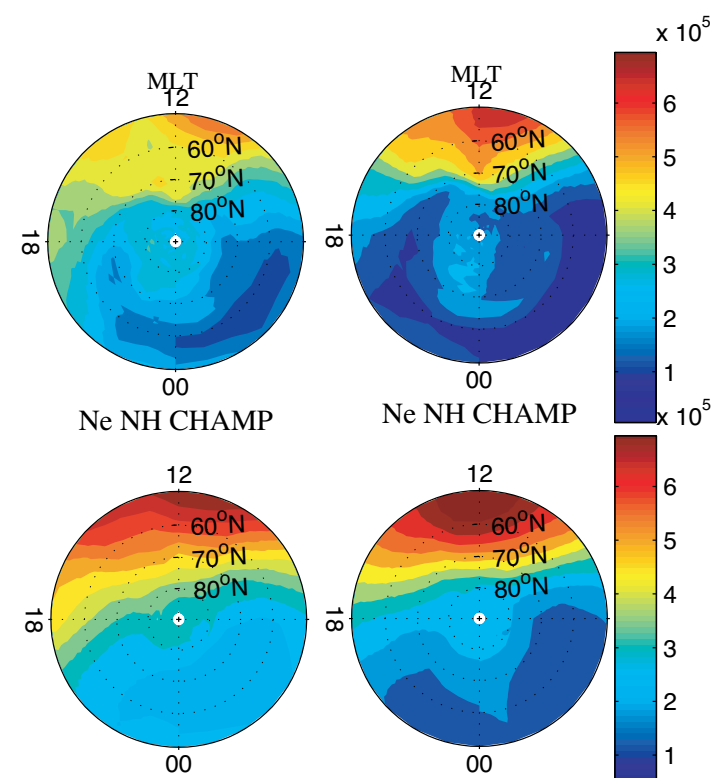

$\mathrm{Ne}$ NH IRI

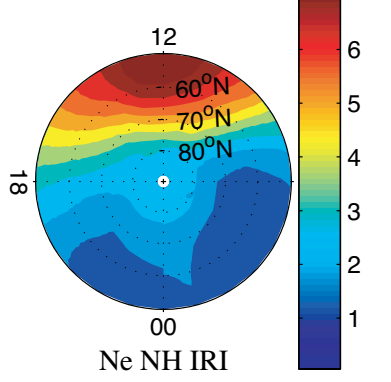

Ne NH IRI
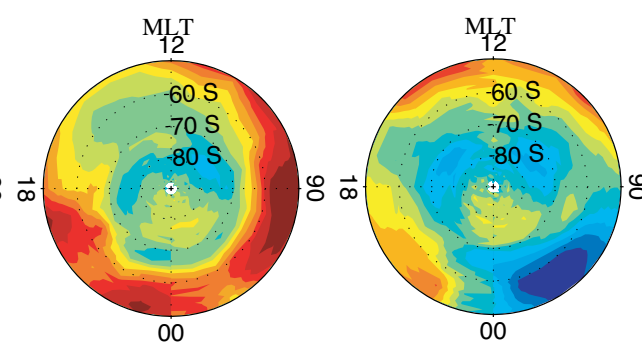

$\mathrm{Ne}$ SH CHAMP

$\mathrm{Ne}$ SH CHAMP

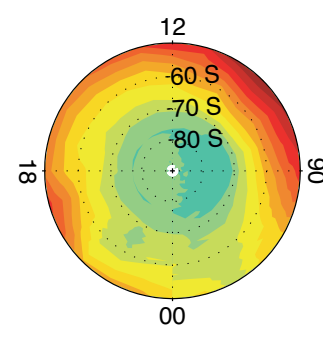

$\mathrm{Ne}$ SH IRI

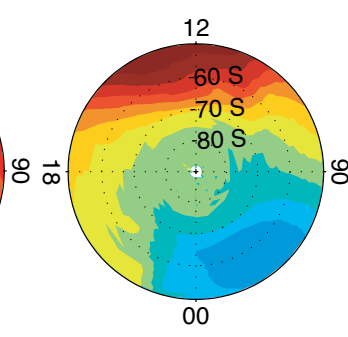

Ne SH IRI
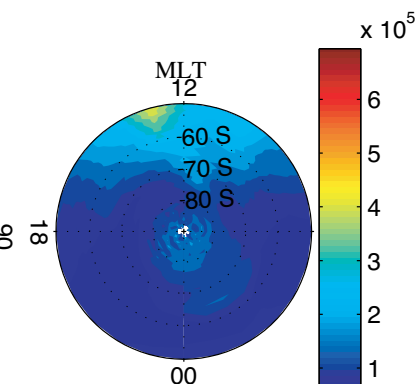

Ne SH CHAMP

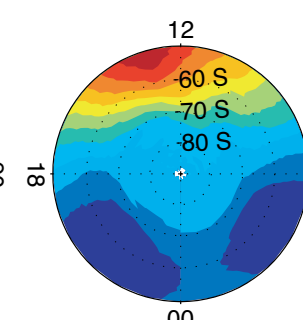

Ne SH IRI

Figure 1. The distribution of the electron density (in unit of $\mathrm{cm}^{-3}$ ) at $400 \mathrm{~km}$ altitude in different seasons from CHAMP and IRI, for quiet $(\mathrm{Kp}=0 \ldots 2)$ geomagnetic conditions. The upper two rows are for the northern hemisphere and the lower two for the southern hemisphere. Columns from left to right are for local seasons: spring, summer, autumn, and winter. 


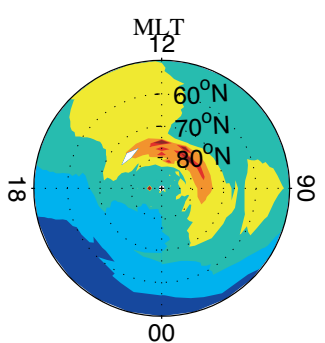

Te NH CHAMP

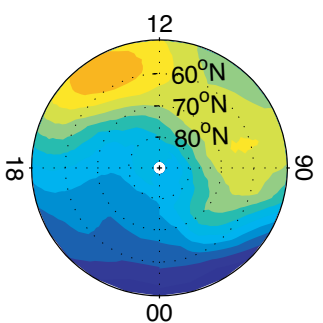

Te NH IRI

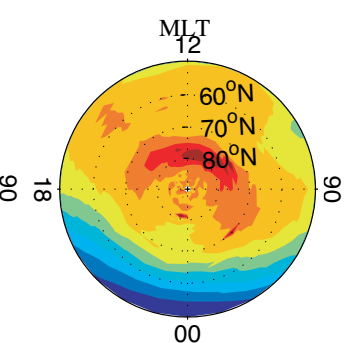

Te NH CHAMP

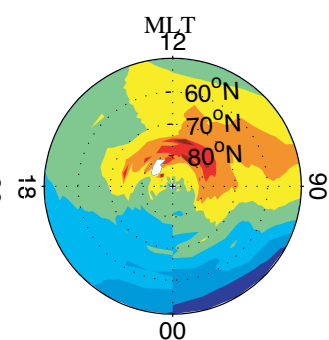

Te NH CHAMP

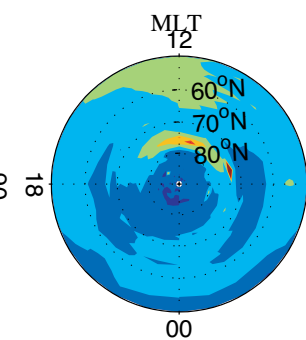

Te NH CHAMP

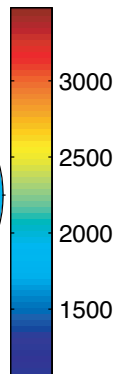

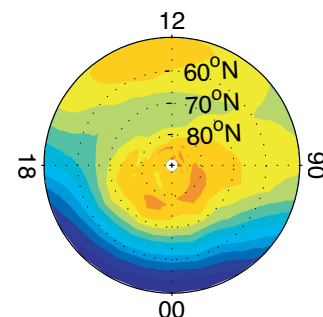

Te NH IRI

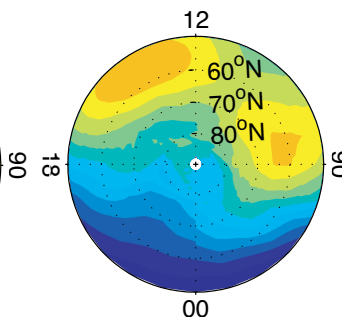

Te NH IRI

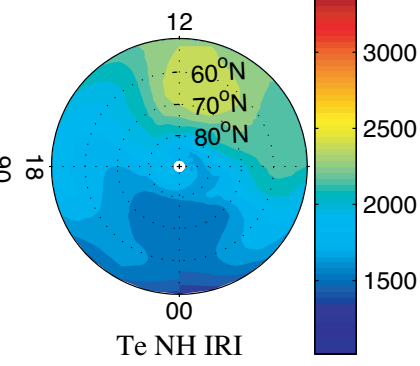

Figure 2. The quiet-time distribution of the electron temperature (in unit of K) at $400 \mathrm{~km}$ altitude in northern hemisphere for different seasons. Columns from left to right are: spring, summer, autumn, and winter.

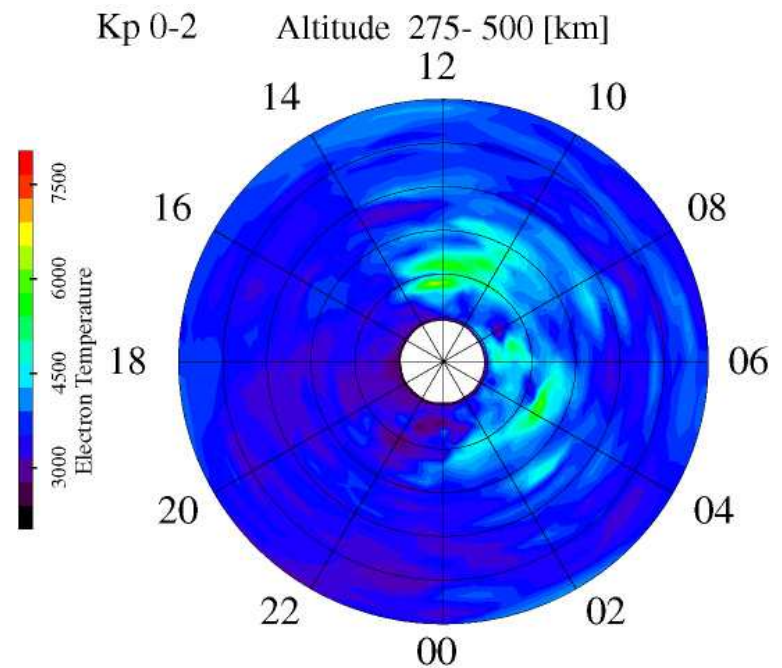

Figure 3. Seasonally averaged electron temperature (in unit of K) between 275-500 $\mathrm{km}$ altitudes observed by Akebono satellite. The latitudes starting from inner most circle are $85^{\circ} \mathrm{N}, 80^{\circ} \mathrm{N}, 75^{\circ} \mathrm{N}$, etc. 


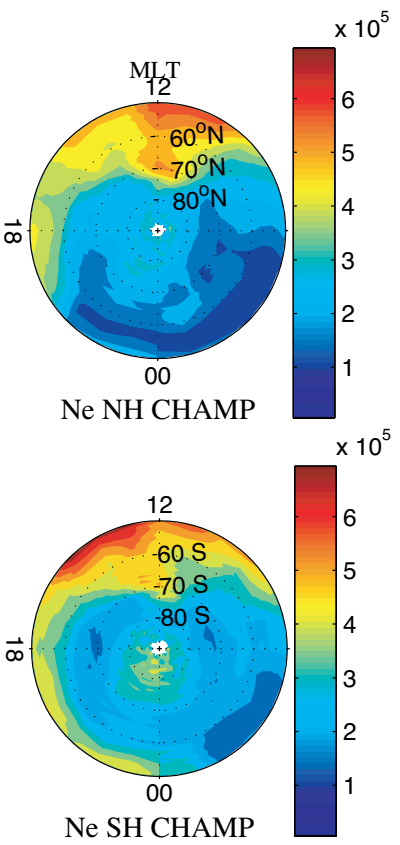

Figure 4. The distribution of the electron density (in unit of $\mathrm{cm}^{-3}$ ) at $400 \mathrm{~km}$ altitude in local autumn observed by CHAMP under moderate geomagnetic conditions $(\mathrm{Kp}=3 \ldots 4+)$. 


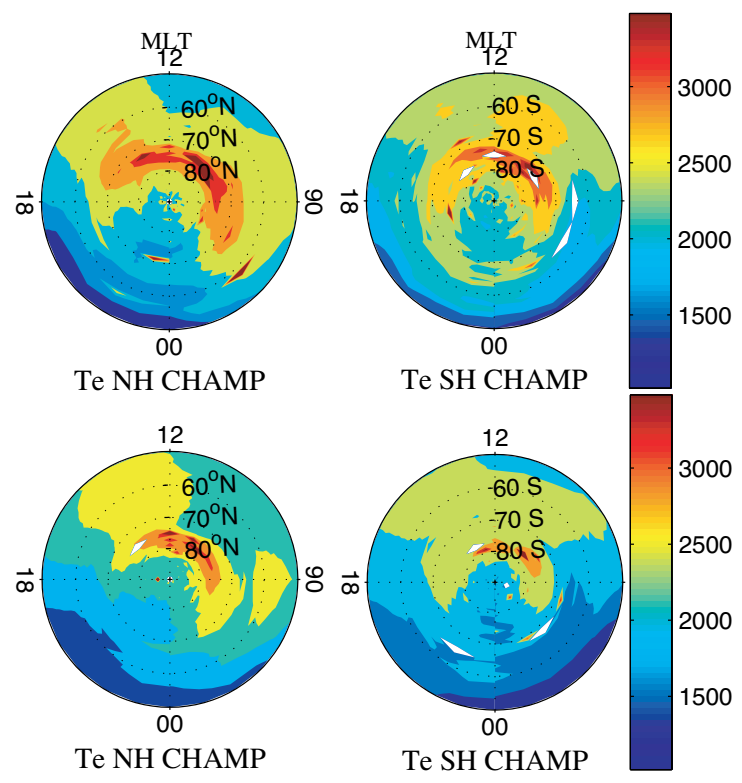

Figure 5. The distribution of the electron temperature (in unit of $\mathrm{K}$ ) at $400 \mathrm{~km}$ altitude in local spring observed by CHAMP under moderate (plates in the upper row) and quiet (plates in the lower row) geomagnetic conditions. 

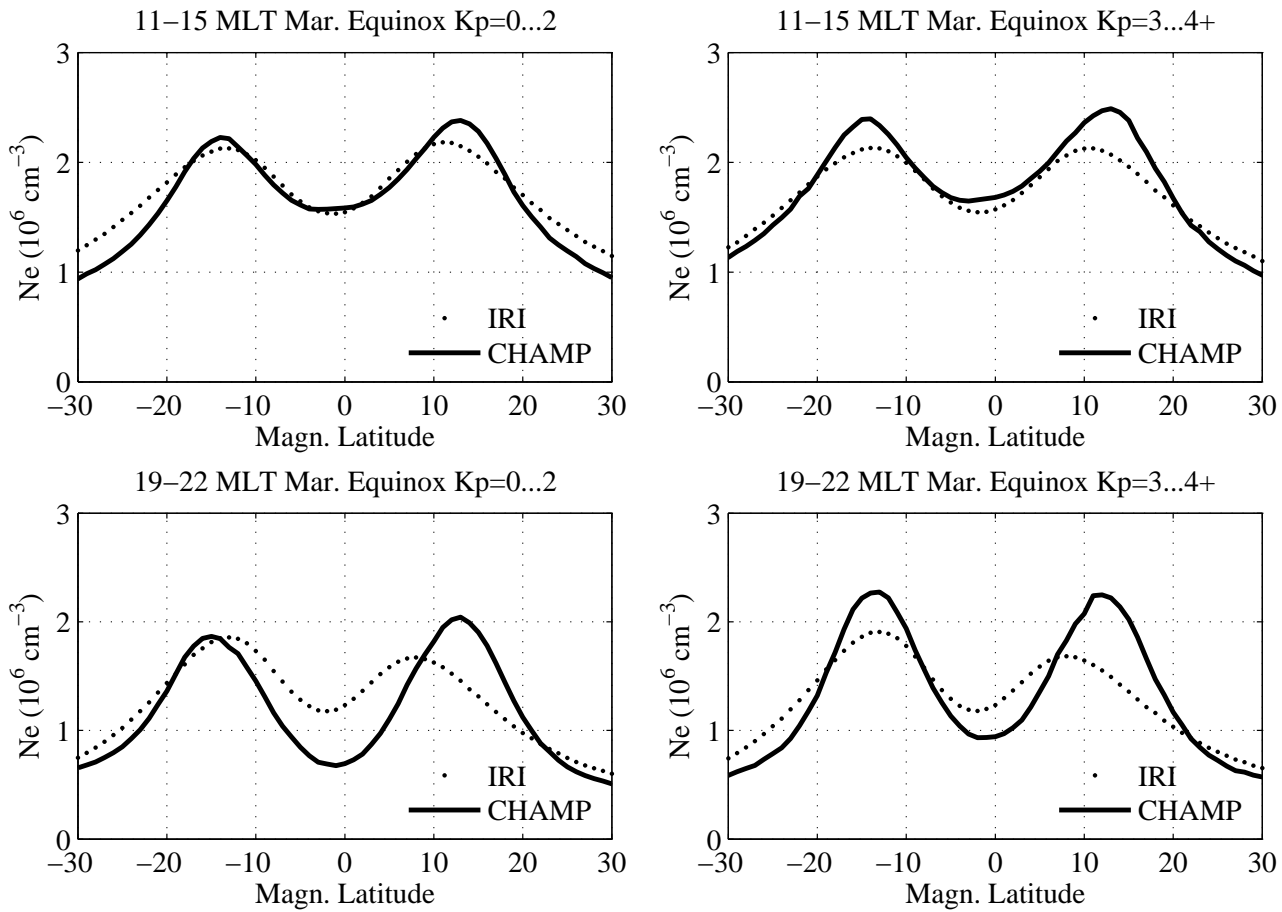

Figure 6. The latitudinal distribution of the electron density at $400 \mathrm{~km}$ altitude around March Equinox for F10.7=150 condition but at different geomagnetic activity levels.

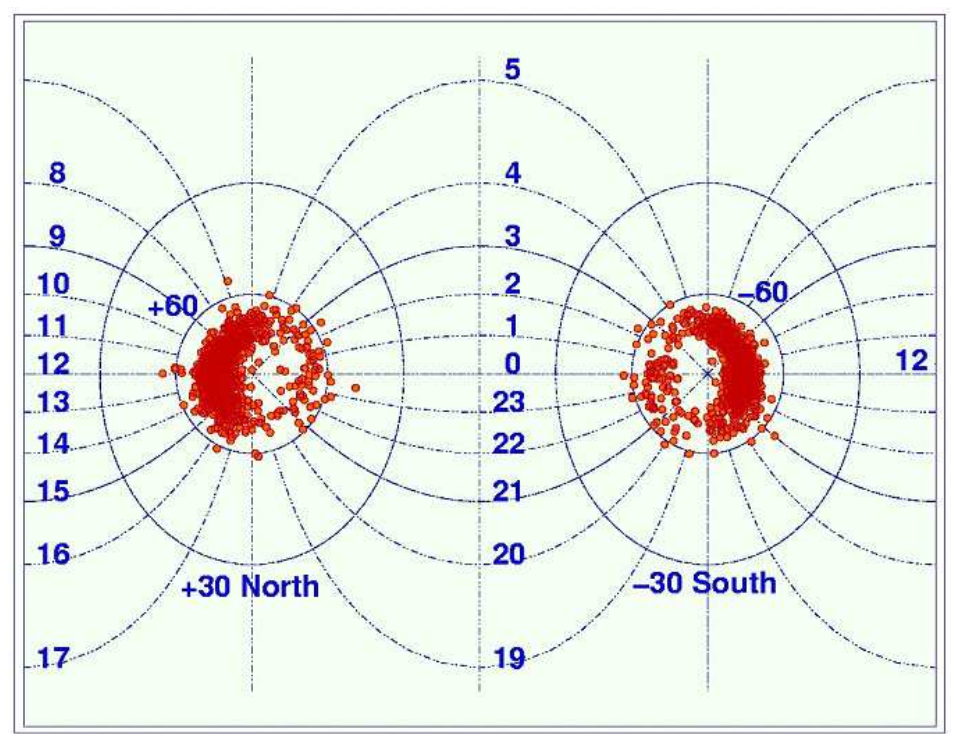

Figure 7. The distribution of small-scale FACs over geomagnetic latitudes and local time for both hemispheres observed by CHAMP. 